\title{
Bronchiectasis in children with primary immune deficiency diseases
}

\author{
ALEKSANDRA SZCZAWIŃSKA-POPŁONYK', KATARZYNA JONCCZYK-POTOCZNA², \\ ZDZISEAWA KYCLER', ANNA BRĘBOROWICZ ${ }^{1}$
}

${ }^{1}$ Department of Pediatric Pneumonology, Allergology and Clinical Immunology, Poznan University of Medical Sciences, Poznan, Poland ${ }^{2}$ Department of Pediatric Radiology, Poznan University of Medical Sciences, Poznan, Poland

\begin{abstract}
Background: Primary immune deficiencies are a common cause of bronchiectasis in children.

Aim of the study: The aim of this study was to evaluate the incidence and characteristic radiological findings of bronchiectasis in children with primary immune deficiencies.

Material and methods: A retrospective review of medical records and pulmonary imaging (chest radiographs and high resolution computed tomography scans) of forty-one patients with primary immune deficiency diseases, aged between 6 months and 18 years was undertaken.

Results: Based on high resolution computer tomography (HRCT) imaging, the diagnosis of bronchiectasis was established in 5 patients (12.2\%), with Bruton's agammaglobulinemia (XLA), common variable immunodeficiency (CVID) and the hyperimmunoglobulin E syndrome (HIES). In 3 children (7.2\%), two with IgG subclass deficiencies (IgGsD) and in one with HIES, pre-bronchiectasis was demonstrat$e d$. The HRCT features of bronchiectasis were predominatingly detected in the basilar segments of the lower lobes, followed by the right middle lobe and the lingula. The median age of 5 children with bronchiectasis visualized in HRCT imaging was 13 years. Pyogenic bacteria were recovered in the respiratory tract of all the children with bronchiectasis, with Streptococcus pneumoniae most frequently identified.

Conclusions: Bronchiectasis is a frequent pulmonary complication and should be actively looked for in children with primary immune deficiencies and lung infections.
\end{abstract}

Key words: bronchiectasis, children, immune deficiency.

(Centr Eur J Immunol 2012; 37 (4): 371-377)

\section{Introduction}

Primary immune deficiencies (PIDs) are a heterogeneous group of genetically determined disorders of the structure of the organs, as well as of differentiation and maturation of the cells of the immune system. Primary immune deficiencies in children pose a high risk of a chronic airway disease and pulmonary complications. Development of bronchiectasis has been linked particularly with a group of primary antibody production defects, involving an absence or reduced levels of one or more immunoglobulin isotypes or subclasses and/or antibodies against specific pathogens, such as X-linked agammaglobulinemia, common variable immunodeficiency [1], hyper-IgM syndrome [2], IgA deficiency, IgG subclass deficiency and specific polysaccharide antibody deficiency [3, 4]. Hypogammaglobulinemia posing the risk of bronchiectasis may also be a manifestation of syndromic immunodeficiencies (previously termed as "other well-defined immunodeficiency syndromes"), including ataxia-telangiectasia [5, 6], as well as DiGeorge syndrome [7] and hyperimmunoglobulin E syndrome $[8,9]$.

The antibody production defects typically predispose affected patients to bacterial pyogenic infections, most frequently with encapsulated bacteria, such as Streptococcus pneumoniae, Haemophilus influenzae, as well as with other Gram positive and Gram negative bacterial pathogens, namely Staphylococcus aureus, Moraxella catarrhalis and Pseudomonas aeruginosa. Opportunistic infections with Pneumocystis jiroveci or invasive fungi, albeit less typical 
for humoral PIDs, may also be present, if T-cell depletion or dysfunction coexists. Furthermore, respiratory viruses, particularly rhinovirus, proved to be a frequent cause of infection in this group of pediatric PIDs, as was demonstrated by Kainulainen et al. [10].

Bronchiectasis is defined by the presence of an abnormal, permanent and progressive dilation of the bronchi. Its pathogenesis in immunodeficiency diseases reflects the Cole's hypothesis of a "vicious circle" of infection and inflammation, in which genetic susceptibility resulting from a defect in the host's defense is a background to the bacterial infection of the airways leading to a chronic progressive inflammatory reaction and lung damage [11]. The inflammatory infiltrations in bronchiectasis involve T lymphocytes, macrophages and neutrophils, most prominent in the airway lumen, which release mediators, such as proteases and elastase, contributing to bronchial dilation. Macrophages and lymphocytes are predominating cell types of infiltrates involving the bronchial wall. These cells are likely to form lymphoid follicles, reflecting the inflammatory process in the small airways and, with progression of the disease, leading to the airflow obstruction and the spreading of the inflammation beyond the airways to the adjacent parenchyma, to cause interstitial pneumonia [12].

High resolution computed tomography (HRCT) scanning is currently a "golden standard" for diagnosing bronchiectasis in children as a highly sensitive, non-invasive imaging modality. The main diagnostic HRCT criteria of bronchiectasis are the following: 1) cross sectional internal diameter of a bronchus is wider than that of its accompanying pulmonary artery (the "signet ring" sign); 2) nontapering bronchi (dilated and thickened airways, the "tram track" appearance); 3) bronchi visible in the outer 1-2 cm of the lung fields, adjacent to the non-mediastinal pleura [13].
The recognition of the characteristic features of bronchiectasis in thoracic imaging is necessary in order to differentiate between the many possible causes of chronic airway, parenchymal and interstitial pulmonary diseases as well as mediastinal disorders. The early diagnosis of bronchiectasis in immunodeficient pediatric patients may contribute to the choice of the optimal therapeutic option and the evaluation of its gravity may influence the prognosis.

\section{Aim of the study}

To determine the incidence and the characteristic radiographic and HRCT findings of bronchiectasis in children with primary immune deficiencies associated with lower respiratory tract infections.

\section{Material and methods}

Participants were recruited at the clinical immunology department of the university hospital. The cases consisted of 41 patients, 25 boys and 16 girls, aged from 6 months to 18 years at the time of the radiological examination (the current median age estimated for specific diagnoses being 10 years old). In all the participating children, diagnoses of primary immunodeficiency diseases had been established, as shown in Table 1. The chest X-ray (CXR) and the HRCT imaging were carried out at the department of pediatric radiology.

This study was a retrospective analysis of radiographic and HRCT findings assessed in children with PIDs and a secondary evaluation of the characteristic features of bronchiectasis in affected individuals. The suspicion of bronchiectasis based on clinical symptoms and radiographic manifestation was confirmed radiologically by HRCT imag-

Table 1. Data of participating children with primary immunodeficiencies

\begin{tabular}{|c|c|c|}
\hline Diagnosis & Number of patients (gender) & Median age at CXR/HRCT \\
\hline agammaglobulinemia X-linked & 4 (boys) & 7 years \\
\hline common variable immunodeficiency & 7 (6 boys, 1 girl) & 14 years \\
\hline hyper-IgM syndrome & 1 (girl) & 15 years \\
\hline IgG3 subclass deficiency & 10 ( 7 boys, 3 girls $)$ & 7 years \\
\hline hypoimmunoglobulinemia M & 3 (1 boy, 2 girls) & 3 years \\
\hline transient hypogammaglobulinemia of infancy & 3 (2 boys, 1 girl) & 6 months \\
\hline DiGeorge syndrome & 2 (girls) & 10 months \\
\hline hyper-IgE syndrome & 3 (girls) & 13 years \\
\hline Nijmegen breakage syndrome & 3 ( 2 boys, 1 girl) & 13 years \\
\hline ataxia-telangiectasia & 2 (2 boys) & 18 years \\
\hline Griscelli syndrome type 2 & 2 (girls) & 12 years \\
\hline severe combined immunodeficiency X-linked & 1 (boy) & 2 years \\
\hline
\end{tabular}


ing with a contrast medium. Children affected with syndromic immunodeficiencies associated with radiosensitivity (ataxia-telangiectasia, Nijmegen breakage syndrome) were not directed to HRCT examination which is admittedly a sensitive marker of the lung structure, but also leads to the inherent exposure to radiation. In a group of children with other PID diagnoses and without lower airways infection or parenchymal lung disease, in whom chest radiographic examinations did not show either any abnormalities or the inflammatory changes were confined to mild bronchopneumonia, HRCT imaging was not indicated. In children with bronchiectasis identified on radiological features, the analysis of the results of sputum cultures was also performed. Older children were able to expectorate, whereas in the infant a post-tussive suction specimen was obtained for microbiologic examination.

\section{Results}

In total, 41 chest radiographs and $21 \mathrm{CT}$ scans were evaluated. In any of the evaluated cases, the chest radiographic examination was not suggestive of bronchiectasis and the clinical symptoms of chronic lung disease were implications for the further evaluation with high-resolution computed tomography. Based on HRCT imaging, the diagnosis of bronchiectasis was established in 5 patients (12.2\%) of the total 41 cases studied, with the following diagnoses: Bruton's agammaglobulinemia (XLA) (2 children), common variable immunodeficiency (CVID) (2 children) and the hyperimmunoglobulin E syndrome(HIES) (1 child). In the next 3 children (7.2\%), in two with IgG subclass deficiencies (IgGsD) and in one with HIES, bronchial dilation considered as pre-bronchiectasis was observed. The HRCT features of bronchiectasis, such as bronchial wall thickening and the lack of tapering of the distal bronchial portions were predominately detected in the basilar segments of the lower lobes, followed by the right middle lobe and the lingula (Fig. 1A, B, 2A, B, 3A-C).

The median age of 5 children with bronchiectasis visualized in HRCT imaging was 13 years old, ranging between 7 and 17 years. The youngest patient with enlarged bronchial lumen on the CT scans was only 9 months old and since the early infancy he suffered from recurrent lower respiratory tract infections. The diagnosis in this patient was IgG3 subclass deficiency; however further monitoring is necessary in this case because he is still at risk of development of a more serious antibody production defect, such as CVID.

The characteristics of patients with bronchiectasis, diagnoses of PIDs and radiological findings evaluated on CXR as well as HRCT examinations are displayed in Table 2.

In this study, albeit limited by a small number of children with pre- and bronchiectasis (together 8 cases of total 41 participants, $19.5 \%$ ), this particular airway pathology was most frequently detected in children with primary antibody

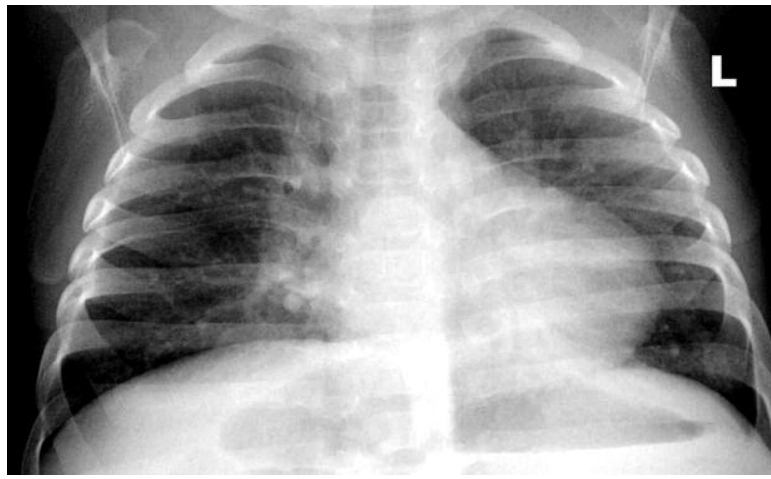

Fig. 1A. Postero-anterior chest radiograph. Peribronchial consolidations in the central portions of the lungs - bronchopneumonia

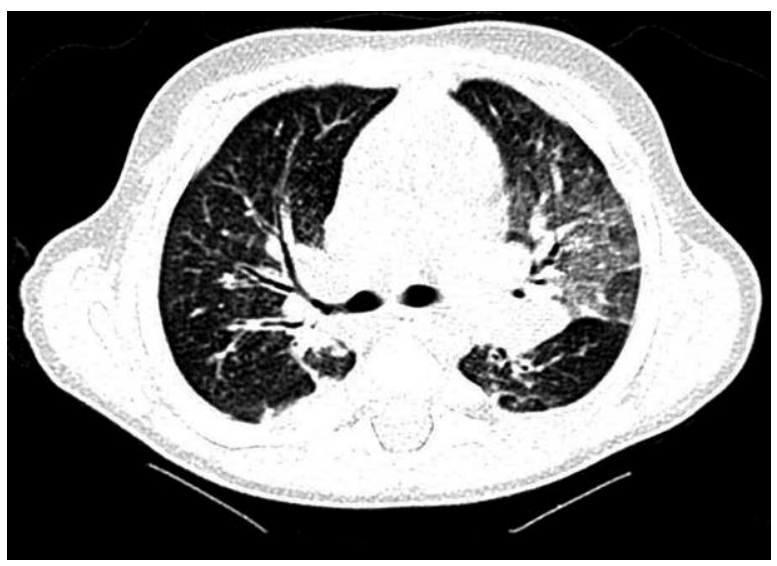

Fig. 1B. CT scan chest image. Dilation of the proximal bronchial segments of the upper lobes, with thickening of the peribronchium. In the upper lobes, in the left segments $1+2$ visible areas of "white glass" sign and bilaterally, parenchymal densities with atelectasis in the right and left segments 6

production defects - Bruton's agammaglobulinemia, common variable immunodeficiency and IgG3 subclass deficiency (together 6 children, accounting for $75 \%$ of cases).

Sputum cultures revealed bacterial pathogens in the respiratory tract of all children with bronchiectasis, with Streptococcus pneumoniae most frequently recovered (in 5 cases), followed by Haemophilus influenzae and Pseudomonas aeruginosa (both pathogens isolated in 2 children).

Of these children with humoral immune deficiencies, 5 of them were treated with polyvalent immunoglobulins in monthly intravenous transfusions (IVIG). The youngest patient in our study group, a 9 month old infant with IgG3 subclass deficiency and pre-bronchiectasis stage evaluated in chest HRCT received 6-week lasting antibiotic therapy. A chronic suppurative disease of the airways characterizing the hyperimmunoglobulin E syndrome manifested as bronchiectasis in one case; in the other one an initial stage, 


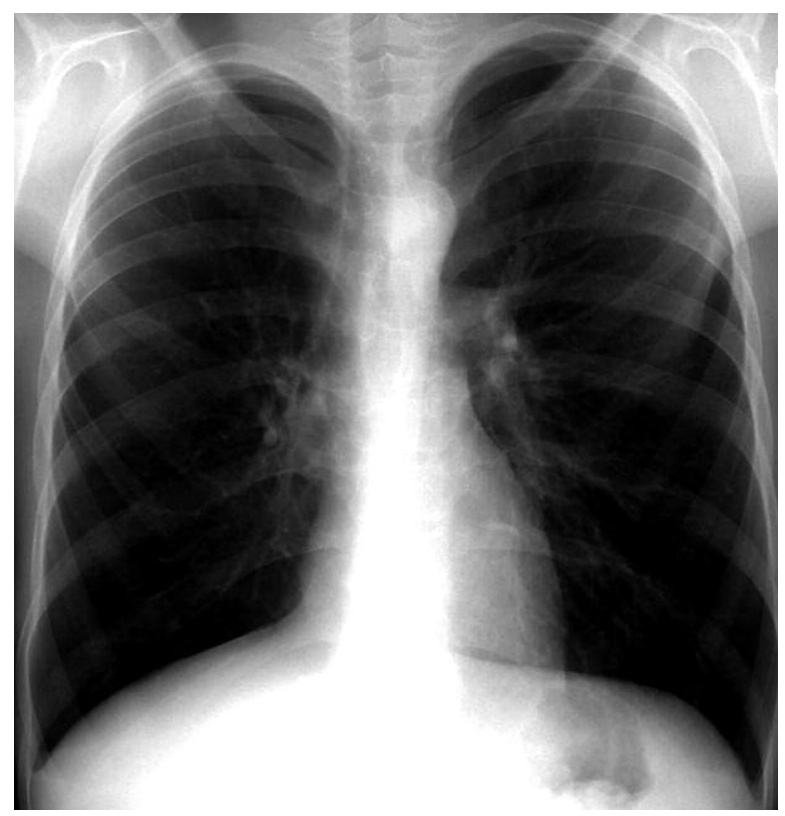

Fig. 2A. Postero-anterior chest radiograph. Inconsiderable peribronchial consolidation in the left lower lung field

pre-bronchiectasis was observed. Both HIES patients were treated with IVIG on clinical indications.

\section{Discussion}

The most common conditions associated with the development of childhood are respiratory infections. Albeit considered a rare disorder in children in the developed world, but radiologically defined pediatric non-cystic fibrosis bronchiectasis is not uncommon. Primary immunodeficiency resulting in a predisposition toward chronic parenchymal and airway infectious pathology is a significant cause [14]. In a study by Eastham et al. [13], the association of non-cystic fibrosis bronchiectasis with immunodeficiency was found in $21 \%$ of a total of 93 children studied. Likewise, immunodeficiency was diagnosed in $17 \%$ of 23 children with bronchiectasis, reported by Karakoc et al. [15] and even in $33 \%$ of the 136 children with this airway pathology evaluated in the study by Li et al. [16]. In a retrospective review of a group of 18 pediatric patients with bronchiectasis related to primary immunodeficiency diseases reported by Haidopoulou et al. [14] the most common PID diagnosis was common variable immunodeficiency (in $33 \%$ of cases), followed by X-linked agammaglobulinemia. Immunoglobulin $\mathrm{G}$ subclass deficiency was found in $11 \%$ of the total number of patients in this study and in $8 \%$ of children with PIDs and chronic sinopulmonary infections evaluated by Ozkan et al. [3]. However, the isolated IgG subclass deficiency as a cause of bronchiectasis in the setting of normal total serum IgG concentration remains controversial and has been proved to coexist with an impaired

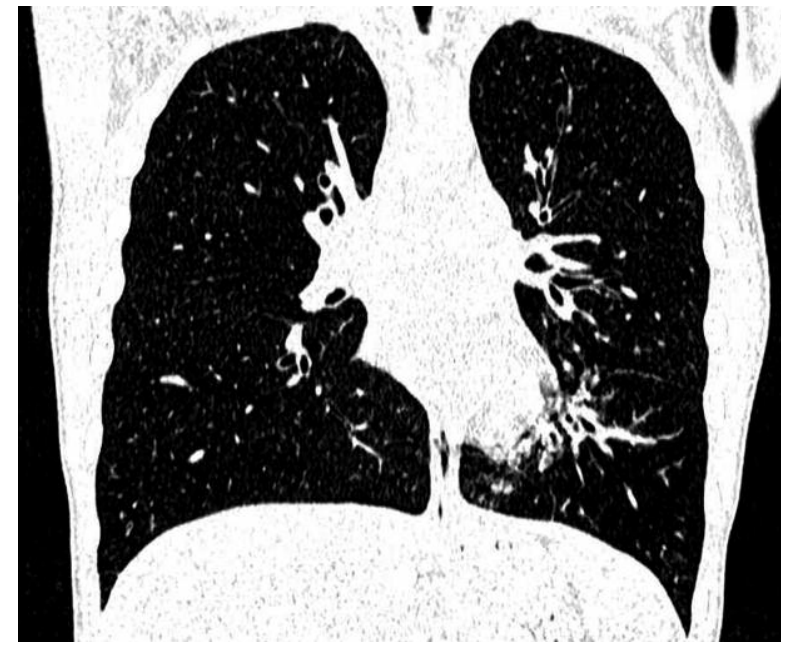

Fig. 2B. CT scan chest image, frontal section. Bronchiectasis in the segments of the right middle lobe and in the segments of the lingual and of the left lower lobe

specific antipolysaccharide antibody response [17]. In contrast to the above mentioned reports where patients with bronchiectasis were evaluated towards underlying conditions and the airway pathology preceded the diagnosis of the immunodeficiency state, in all 8 children with bronchiectasis who participated in our study, the diagnosis of primary immunodeficiency had been established prior to the evaluation of the pathology of the airway. Bacterial pathogens were identified in the airways of all immunodeficient children with bronchiectasis and the most common isolates were Streptococcus pneumoniae, followed by Haemophilus influenzae and Pseudomonas aeruginosa, with the first microorganism associated with the most severe bronchiectasis. Similar results from sputum cultures from patients with non-cystic fibrosis bronchiectasis were reported in other investigations [18].

In none of the cases with bronchiectasis or a prebronchiectasis state, the chest radiograph was not suggestive and thus not helpful in establishing the diagnosis of the airway injury. In all of these children, persistent respiratory symptoms suggested the need for further investigations and HRCT chest imaging. The discrepancy between CXR and HRCT in diagnosing bronchiectasis in our study referred to $100 \%$ of patients. These data are consistent with the results of other clinical studies. In the above mentioned clinical and radiological review of 93 children with bronchiectasis of various underlying etiology performed by Eastham et al. [13], the agreement between the chest radiograph and HRCT scan for diagnosis of bronchiectasis was found in only $5 \%$ of cases. Therefore, these findings suggest that the chest radiograph is of little diagnostic value for diagnosing bronchiectasis in children and a normal CXR should not preclude further investigation with HRCT undertaken on clinical grounds. 


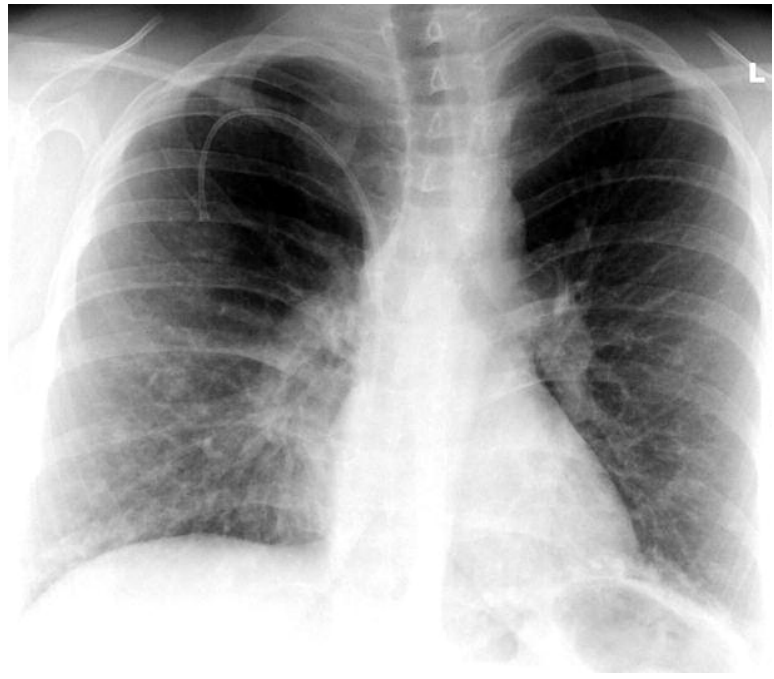

Fig. 3A. Postero-anterior chest radiograph. Bilateral interstitial consolidations in the lower lung fields accompanied by fine patchy parenchymal infiltrations in the left lower lobe

Radiographic features in the patients studied who were associated with bronchiectasis or a pre-bronchiectasis state included: parenchymal consolidations accompanied by interstitial opacities found in 4 children, followed by peribronchial consolidations observed in 3 children and findings reflecting advanced parenchymal and airway disease with hyperinflation, foci of emphysema and fibrosis were seen in 1 child; a normal CXR was seen in 1 child with bronchiectasis visualized in HRCT imaging (data displayed in Table 2). Likewise, Redding and co-workers who evaluated a group of 10 children with bronchiectasis, demonstrated that the lung and hence airway injury which was manifested in chest radiographs predominantly as parenchymal densities or pneumonia rather than hyperinflation or atelectasis [19]. The radiological extent of bronchiectasis was the greatest in the left lower lobe $(n=5,62.5 \%)$, followed by the right lower lobe $(n=4,50 \%)$ and this localization mostly correlated with the diagnosis of profound antibody production defects, CVID and XLA; equally involved (in 2 cases, 25\%, respectively) subsequently were the right middle lobe, the right upper lobe and the left upper lobe. In 6 of total number of $8(75 \%)$ children with bronchiectasis or a pre-bronchiectasis state, two lobes were involved; a single left lower lobe was affected with bronchiectasis in one child and with pre-bronchiectasis in an another. Similar data regarding the widespread and severity of bronchiectasis were reported by Edwards et al. [20] who demonstrated the bilateral disease in $91 \%$ of children, with a predominating involvement of the left lower lobe.

In our study, the patients' mean age on the diagnosis of bronchiectasis was 9 years with the age range from 3 years in a patient with CVID to 15 years in a child with HIES; however it is worth noting that the youngest patient with pre-

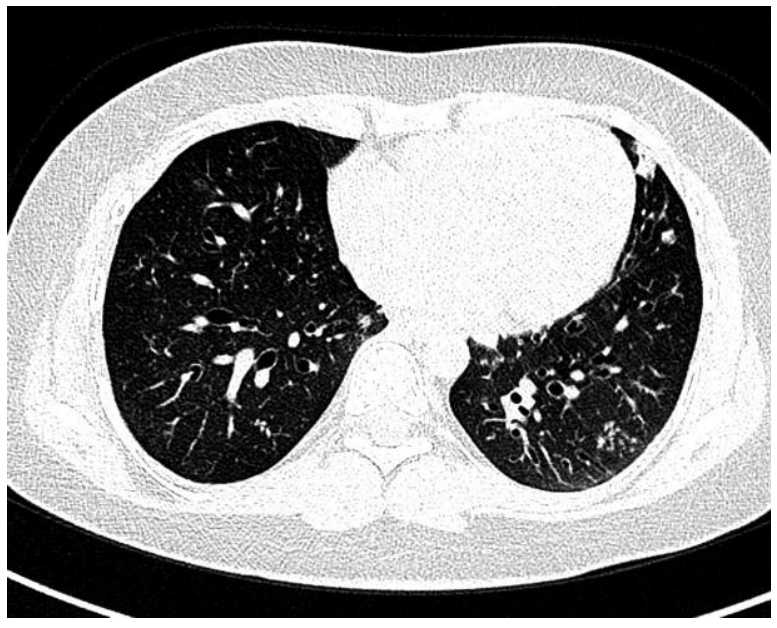

Fig. 3B. CT scan chest image, transverse section. Bronchiectasis in the basilar segments of both lungs; besides, in the segment 9 of the left lung fine nodular consolidations are visible - "tree in bud" pattern

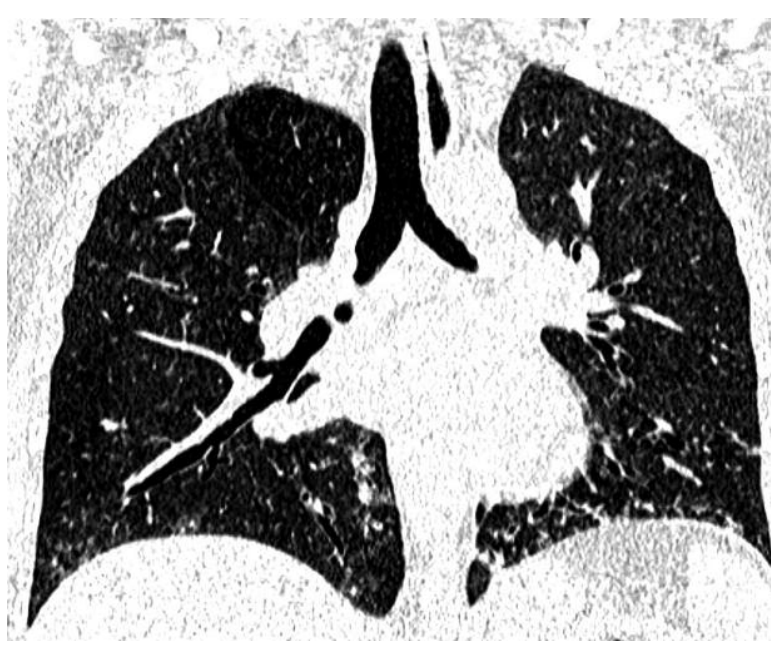

Fig. 3C. CT scan chest image, frontal section. Bronchiectasis in the basilar segments of both lungs and fine nodular consolidations - "tree in bud"

bronchiectasis, in whom IgG3sD was identified, was only 9 month old. Hence, the age of diagnosis of the bronchial disease did not correlate either with age or with specific diagnosis of primary immunodeficiency. Nevertheless, the overall tendency among the children studied who had PIDs was to develop bronchiectasis later than in patient groups reported by other authors, who identified this airway pathology in children at a mean age between 3 years $[19,20]$ and 7 years [13]. It may be presumed that establishing the diagnosis of primary immunodeficiency and early institution of specific treatment with intravenous immunoglobulins and/or antibiotic therapy contributed to a slowdown in the airway and lung injury in our children. However, taking into consider- 
Table 2. Characteristics of patients with bronchiectasis and radiological findings

\begin{tabular}{|c|c|c|c|c|c|}
\hline $\begin{array}{l}\text { Patient } \\
\text { ID }\end{array}$ & Sex & $\begin{array}{l}\text { Diagnosis } \\
\text { of PID }\end{array}$ & $\begin{array}{c}\text { Age at } \\
\text { diagnosis of } \\
\text { bronchiectasis }\end{array}$ & CXR findings & HRCT findings \\
\hline 1 & M & XLA & 10 years & $\begin{array}{l}\text { Irregular strands of parenchymal consolidations } \\
\text { alongside bronchi and vessels in the basilar } \\
\text { posterior segment of the left lower lobe; in their } \\
\text { surroundings fine alveolar foci of emphysema } \\
\text { and linear fibrosis; hyperinflation of the distal } \\
\text { portions of both lungs }\end{array}$ & $\begin{array}{l}\text { Cylindrical bronchiectasis in basilar } \\
\text { segments of the left lung }\end{array}$ \\
\hline 2 & M & XLA & 5 years & $\begin{array}{l}\text { Parenchymal and interstitial opacities in the } \\
\text { upper and lower fields of the right lung }\end{array}$ & $\begin{array}{l}\text { Cylindrical bronchiectasis in the segment } \\
3 \text { and bronchial dilation in segments } \\
7 \text { and } 8 \text { of the right lung }\end{array}$ \\
\hline 3 & M & CVID & 12 years & Lung fields without consolidations & $\begin{array}{l}\text { Cylindrical bronchiectasis in the right } \\
\text { middle lobe, in the lingual and in the left } \\
\text { lower lobe }\end{array}$ \\
\hline 4 & M & CVID & 3 years & $\begin{array}{l}\text { Parenchymal and interstitial opacities in the right } \\
\text { lower lobe and in the lingula }\end{array}$ & $\begin{array}{l}\text { Cylindrical bronchiectasis in the right and } \\
\text { left lower lobes }\end{array}$ \\
\hline 5 & $\mathrm{~F}$ & HIES & 15 years & $\begin{array}{l}\text { Interstitial opacities in the lower fields of both } \\
\text { lungs accompanied by patchy parenchymal con- } \\
\text { solidations in the left lower lobe }\end{array}$ & $\begin{array}{l}\text { Cylindrical bronchiectasis in basilar segments } \\
\text { of both lungs accompanied by nodular den } \\
\text { sities, "tree in bud" pattern }\end{array}$ \\
\hline 6 & $\mathrm{~F}$ & HIES & 14 years & $\begin{array}{l}\text { Peribronchial consolidations in the perihilar re- } \\
\text { gions of both lungs and in the right lower lobe }\end{array}$ & $\begin{array}{l}\text { Enlargement of the bronchial lumen in } \\
\text { the left lower lobe (pre-bronchiectasis) }\end{array}$ \\
\hline 7 & M & IgG3sD & 14 years & $\begin{array}{l}\text { Peribronchial consolidations in the central } \\
\text { portions of both lungs and in the right lower } \\
\text { lobe }\end{array}$ & $\begin{array}{l}\text { Bronchial dilation in the right middle } \\
\text { lobe (segment 5) and in the lingula } \\
\text { (segment 5) (pre-bronchiectasis) }\end{array}$ \\
\hline 8 & M & IgG3sD & 9 months & $\begin{array}{l}\text { Peribronchial consolidations in the central } \\
\text { portions of both lungs }\end{array}$ & $\begin{array}{l}\text { Dilation of the bronchial proximal sections } \\
\text { in the upper lobes (pre-bronchiectasis) with } \\
\text { thickening of the peribronchium; areas of the } \\
\text { "white glass" pattern in the left upper lobe } \\
\text { and bilateral parenchymal consolidations with } \\
\text { atelectasis in segments } 6 \text { of the right and the } \\
\text { left lower lobes }\end{array}$ \\
\hline
\end{tabular}

ation the development of bronchiectasis in a 9-month old infant, we conclude that this bronchial pathology can develop in any group of immunodeficiencies and that eventually does not correlate with either the child's age or the specific diagnosis. Furthermore, the clinical outcome of immune deficiency, particularly of hypogammaglobulinemia in infants and young children is difficult to predict. These children may finally experience a spontaneous immunocorrection as well as may develop a profound immune deficiency such as CVID. Therefore, in each case of bronchiectasis in a pediatric patient, detailed investigation of the immune status needs to be performed.

\section{Conclusions}

Bronchiectasis is a commonly occurring airway pathology among children with primary immune deficiency diseases. In particular, impaired humoral immunity predisposes pediatric patients to bronchial and pulmonary infections, a persistent inflammatory response and chronic airway injury. The lack of consistency between the chest radiograph and a high resolution computed tomography is considerable and the latter examination should be performed and the diagnosis of bronchiectasis actively sought whenever the clinical course of the immunodeficiency and respiratory tract manifestation are suggestive of the pathology.

The authors declare no conflict of interest.

\section{References}

1. Aghamohammadi A, Allahverdi A, Abolhassani H, et al. (2010): Comparison of pulmonary diseases in common variable immunodeficiency and X-linked agammaglobulinemia. Respirology 15: 289-295.

2. Silva R, Da Costa JT (2010): Hyper-IgM syndrome - a case report and a clinical perspective. Eur Ann Allergy Clin Immunol 42: 194-196. 
3. Ozkan H, Atlihan F, Genel F, et al. (2005): IgA and/or IgG subclass deficiency in children with recurrent respiratory infections and its relationship with chronic pulmonary damage. J Investig Allergol Clin Immunol 15: 69-74.

4. Tabatabaie P, Aghamohammadi A, Mamishi S, et al. (2008): Evaluation of humoral immune function in patients with bronchiectasis. Iran J Allergy Asthma Immunol 7: 69-77.

5. McGrath-Morrow SA, Gower WA, Rothblum-Oviatt C, et al. (2010): Evaluation and management of pulmonary disease in ataxia-telangiectasia. Pediatr Pulmonol 45: 847-859.

6. Bott L, Lebreton J, Thumerelle C, et al. (2007): Lung disease in ataxia-telangiectasia. Acta Paediatr 96: 1021-1024.

7. Hong R, Shen V, Rooney C, et al. (2001): Correction of DiGeorge anomaly with EBV-induced lymphoma by transplantation of organ-cultured thymus and Epstein-Barr-specific cytotoxic T lymphocytes. Clin Immunol 98: 54-61.

8. Paulson ML, Freeman AF, Holland SM (2008): Hyper-IgE syndrome: an update on clinical aspects and the role of signal transducer and activator of transcription 3. Curr Opin Allergy Clin Immunol 8: 527-533.

9. Moin M, Farhoudi A, Movahedi M, et al. (2006): The clinical and laboratory survey of Iranian patients with hyper-IgE syndrome. Scand J Infect Dis 38: 898-903.

10. Kainulainen L, Vuorinen T, Rantakokko-Jalava K, et al. (2010): Recurrent and persistent respiratory tract viral infections in patients with primary hypogammaglobulinemia. J Allergy Clin Immunol 126: 120-126.

11. King PT (2009): The pathophysiology of bronchiectasis. Int J Chron Obstruct Pulmon Dis 4: 411-419.

12. King P (2011): Pathogenesis of bronchiectasis. Paediatr Respir Rev 12: 104-110.

13. Eastham KM, Fall AJ, Mitchell L, Spencer DA, et al. (2004): The need to redefine non-cystic fibrosis bronchiectasis in children. Thorax 59: 324-327.

14. Haidopoulou K, Calder A, Jones A, et al. (2009): Bronchiectasis secondary to primary immunodeficiency in children: longitudinal changes in structure and function. Pediatr Pulmonol 44: 669-675.

15. Karakoc GB, Yilmaz M, Altintas DU, Kendirli SG (2001): Bronchiectasis: still a problem. Pediatr Pulmonol 32: 175-178.

16. Li AM, Sonnappa S, Lex C, et al. (2005): Non-CF bronchiectasis: does knowing the aetiology lead to changes in management? Eur Respir J 26: 8-14.

17. Vendrell M, de Gracia J, Rodrigo MJ, et al. (2005): Antibody production deficiency with normal IgG levels in bronchiectasis of unknown etiology. Chest 127: 197-204.

18. Boren EJ, Teuber SS, Gershwin ME (2008): A review of noncystic fibrosis pediatric bronchiectasis. Clin Rev Allerg Immunol 34: 260-272.

19. Redding G, Singleton R, Lewis T, et al. (2004): Early radiographic and clinical features associated with bronchiectasis in children. Pediatr Pulmonol 37: 297-304.

20. Edwards EA, Metcalfe R, Milne DG, et al. (2003): Retrospective review of children presenting with non cystic fibrosis bronchiectasis: HRCT features and clinical relationships. Pediatr Pulmonol 36: 87-93. 